Document downloaded from:

http://hdl.handle.net/10251/78699

This paper must be cited as:

Pérez-Esteve, E.; Ruiz Rico, M.; Torre, CDL.; Llorca Martínez, ME.; Sancenón Galarza, F.; Marcos Martínez, MD.; Amoros Del Toro, PJ.... (2016). Stability of different mesoporous silica particles during an in vitro digestion. Microporous and Mesoporous Materials. 230:196207. doi:10.1016/j.micromeso.2016.05.004.

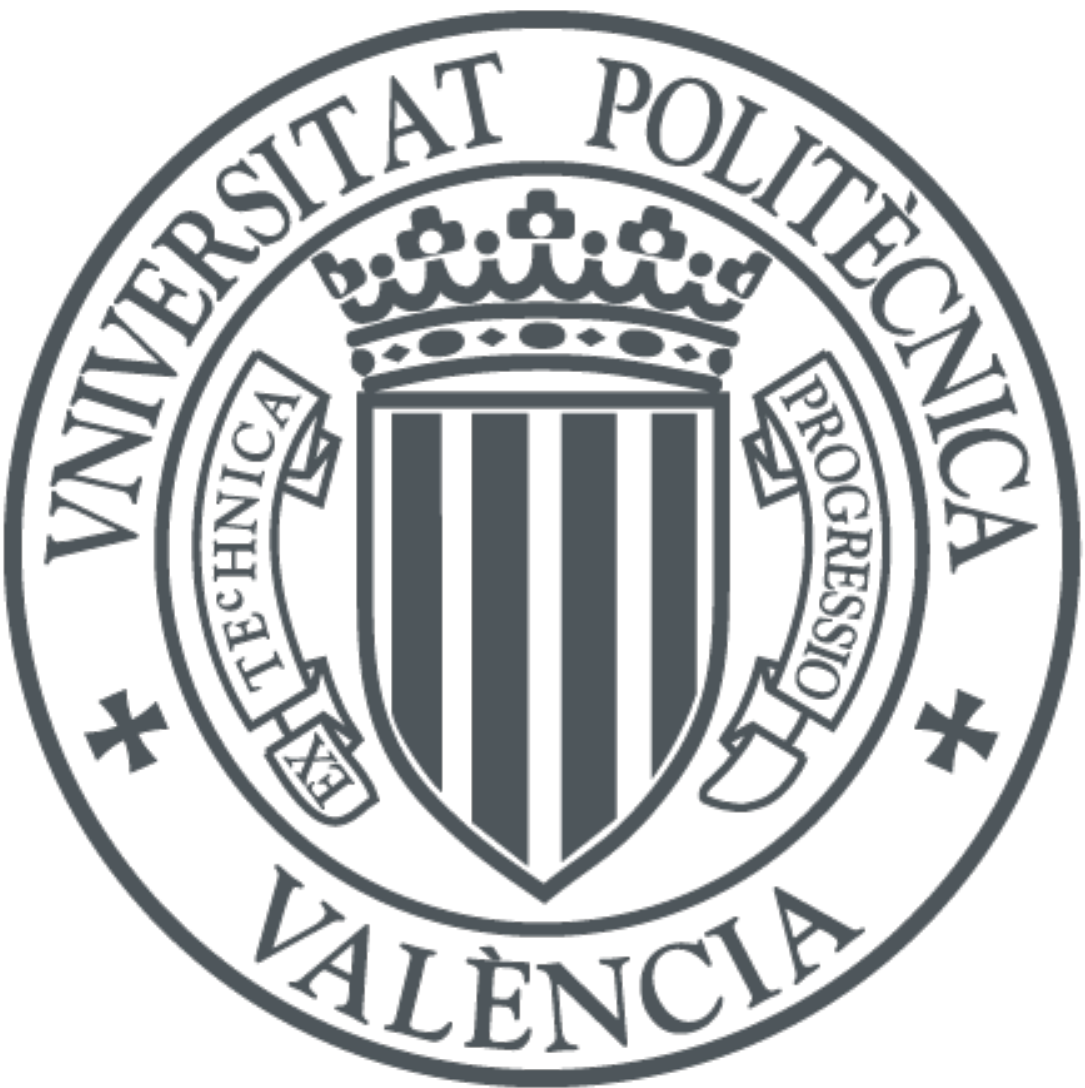

The final publication is available at

http://dx.doi.org/10.1016/j.micromeso.2016.05.004

Copyright Elsevier

Additional Information 


\section{Stability of different mesoporous silica particles during an in vitro}

2

3

4

5

6

7

8

9

\section{digestion}

Édgar Pérez-Esteve ${ }^{a}{ }^{*}$, María Ruiz-Rico $^{a}$, Cristina de la Torre ${ }^{b, c}, E^{2}$ par Llorca ${ }^{d}$, Félix Sancenón ${ }^{b, c}$, María D. Marcos ${ }^{\mathrm{b}, \mathrm{c}}$, Pedro Amorós ${ }^{\mathrm{e}}$, Carmen Guillem ${ }^{\mathrm{e}}$, Ramón Martínez-Máñez ${ }^{\mathrm{b}, c,}$, José Manuel Barat $^{\mathrm{a}}$

${ }^{a}$ Grupo de Investigación e Innovación Alimentaria, Universitat Politècnica de València. Camino de Vera s/n, 46022, Spain

${ }^{\mathrm{b}}$ Instituto Interuniversitario de Investigación de Reconocimiento Molecular y Desarrollo Tecnológico (IDM), Unidad Mixta Universitat Politècnica de València - Universidad de Valencia. Departamento de Química Universitat Politècnica de València, Camino de Vera s/n, 46022, Valencia, Spain ${ }^{\mathrm{C} C I B E R}$ de Bioingeniería, Biomateriales y Nanomedicina (CIBER-BBN)

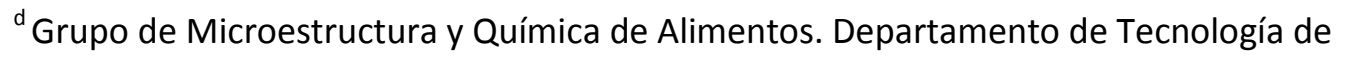
Alimentos, Universitat Politècnica de València. Camino de Vera s/n, 46022, Valencia, Spain e Institut de Ciència dels Materials (ICMUV), Universitat de València, P.O. Box 2085, 46071, Valencia, Spain 
22 biomolecules and can be able to act as smart delivery systems capable to control and target

23 the release of their cargo in a particular part of the gastrointestinal tract when administrated orally. However, the aptness of these encapsulation supports in in vivo oral controlled release relies on their chemical stability through the digestive tube. In this context, we have evaluated the stability of four different mesoporous silica particles, frequently used as encapsulating supports, during an in vitro digestion process comprising buccal, stomach and intestinal phases. Results showed that after $4 \mathrm{~h}$ of digestion, the textural properties of silica supports in the form of nanoparticles (MCM-41 and UVM-7 nanoparticles) were lost in varying degrees, whereas silica microparticles supports (MCM-41 and SBA-15 microparticles) endures better the digestion process. Moreover, the functionalization of the surface with $N^{1}$-(3trimethoxysilylpropyl)diethylenetriamine, an organic moiety commonly used in the preparation of $\mathrm{pH}$-responsive mesoporous silica particles, resulted in an improvement of the stability of the supports.

Keywords: mesoporous silica particles, in vitro digestion, stability, amine-functionalization 


\section{Introduction}

Mesoporous Silica Particles (MSPs) are receiving great attention in the field of oral controlled release due to their capability to improve drug solubility and stability in the gastrointestinal tract (GIT), [1-2] as well as to dosage the cargo along time (sustained controlled release) in specific GIT places (targeted controlled release) [3-5]. These reported features, that convert MSPs in unique smart delivery systems, are due to their large loading capacity [6], low toxicity [7] and the fact that their surface can be functionalized with molecular/supramolecular ensembles. This last feature allows the development of gated-MSPs showing "zero delivery" and capable to release their cargo on-command in response to specifically designated external stimuli [8-10]. Drug delivery/formulation technologies that can improve bioavailability, drug stability and subsequently increase drug effectiveness are much desired in the pharmaceutical sciences [11-12]. In food technology, encapsulation of bioactive molecules (e.g. vitamins, antioxidants, phytochemicals, etc.) may improve their biological stability, facilitate components handling, mask unpleasant sensorial properties and modulate the bioaccessibility of the molecule of interest along the GIT [13].

Besides a high loading capacity, controlled release and biocompatibility, the suitability of MSPs in oral controlled release in in vivo applications depend on the chemical stability of the supports though the whole digestive tube. However, it is known that due to the metastability of MSPs, silica can be biodegraded into silicic acids, including monomeric silicic acid and various polysilicic acids with different polymerization degrees under harsh environments provoking a collapse of the porous structures [14]. In this line, Cauda, Schlossbauer \& Bein studied the biodegradation of colloidal mesoporous silica nanoparticles $(50 \mathrm{~nm})$ in simulated body fluid of bare, globally functionalized, and surface poly(ethylene glycol)-coated colloidal mesoporous silica nanoparticles in simulated body fluid ( $\mathrm{pH}$ 7.4) for a period of 1 month at 37 ${ }^{\circ} \mathrm{C}[15]$. After this period of time, the textural properties of the mesoporous system were lost and pores were blocked because the precipitation of inorganic components from the 
simulated body solution. The stability of the particles increased by surface functionalization with poly(ethylene glycol). The degradation behaviour of surfactant-extracted mesoporous silica in simulated body fluid was also evaluated by He and co-workers proposing a three-stage degradation process comprising a fast bulk degradation on hour-scale, a silicon concentration decrease stage due to a deposition of a calcium/magnesium silicate layer, and a later continuous sustained diffusion beyond days [16]. The same year, Lin, Abadeer \& Haynes, evaluated the stability of small mesoporous silica nanoparticles $(<50 \mathrm{~nm})$ functionalised with poly(ethylene glycol) in $\mathrm{H}_{2} \mathrm{O}$, phosphate buffer solution (PBS) $(\mathrm{pH} 7.5)$, and Dulbecco's modified Eagle's medium (DMEM) with $10 \%$ fetal bovine serum (FBS) ( $\mathrm{pH}$ ca. 7.5) [17]. These particles exhibited long term stability in all these media at both, room and physiological temperature. In a different attempt, El Mourabit et al. studied the stability of mesoporous silica under acidic conditions and a loss of textural properties of the supports was observed [18]. The authors also found that the degradation rate was dependent on the nature of the acidic media (phosphoric acid have stronger impact than hydrochloric or sulphuric acids) and the kind of mesoporous silica used in the study. More recently, Choi et al. studied the biodegradation of SBA-15 in both, simulated body fluid and in vivo [19]. These authors have shown that the degradation rate of SBA-15 was affected by the presence of surface functional groups and synthesis methodologies. Furthermore, in vivo experiment showed that SBA-15 degrade in the animal and pore structure deformation occurs as a function of time.

Most of these studies evaluated the stability of mesoporous silica nanoparticles. However, MSPs can be fabricated with a controlled size from $50 \mathrm{~nm}$ to a few microns. When preparing smart delivery systems based on MSPs, particle size is very important since it conditions the distribution and behavior of particles in living systems. In general, small MSPs can cross epitheliums, can be distributed in the body and be non-specifically internalized by certain cells [20]. In contrary, oversized particles (microparticles) cannot easily cross physical membranes in the body, and thus large particle sizes are preferred for developing orally administrated 
controlled release devices [4]. Having in mind the importance of particle size in oral administration, it may be of importance to study the stability of mesoporous silica with microsized particles. However, stability of large MSPs has been barely studied. Moreover, as far as we know, there are not studies about the effect that the consecutive presence of saliva $(\mathrm{pH}$ 7.5), gastric $(\mathrm{pH} \mathrm{1.2-2)}$ and intestinal fluids $(\mathrm{pH} 7.8-8)$ have on the stability of the small and large MSPs. Thus, notwithstanding the works detailed above, a lack of information about the degradability/stability of MSPs with different sizes during a whole digestion is still unavailable.

The aim of this study was to evaluate the stability of different bare and functionalised mesoporous silica particles differing in particle size, particle shape and pore structure (pore size and wall thickness) during a simulated in vitro digestion. With this purpose a deep evaluation of the stability of textural properties of MSPs during the in vitro digestion was performed. Descriptive studies were completed with the assessment of potential cytotoxicity of digested particles or their degradation products.

\section{Materials and methods}

\subsection{Chemicals}

Tetraethylorthosilicate (TEOS), $N$-cetyltrimethylammonium bromide (CTABr), Pluronic P123 (P123), triethanolamine $\left(\mathrm{TEAH}_{3}\right)$, sodium hydroxide $(\mathrm{NaOH})$, hydrochloric acid $(\mathrm{HCl}), N^{1}-$ (3-trimethoxysilylpropyl)diethylenetriamine (N3), and all chemicals for the preparation of the simulated digestive fluids were provided by Sigma-Aldrich (Poole, Dorset, UK). HPLC grade acetonitrile was provided by Scharlau (Barcelona, Spain). Rhodamine B was acquired from Fluka (Missouri, USA).

For cell culture experiments, trypan blue solution (0.4\%) cell culture grade and dimethyl sulfoxide (DMSO), phosphate buffered saline (PBS) and Dulbecco's Modified Eagle's medium (DMEM) with glucose, L-glutamine and pyruvate for cell culture were provided by Sigma- 
Aldrich (Poole, Dorset, UK). Mc Coy's 5a Medium and Keratinocyte Serum Free Medium, Fetal Bovine Serum (FBS) and trypsin were purchased from Gibco (Life Technologies, Madrid, Spain). Cell proliferation reagent WST-1 was purchased from Roche Applied Science (Barcelona, Spain).

\subsection{Mesoporous silica particles synthesis}

Synthesis of the four different silica particles was carried out following the procedures described previously [4].

MCM-41 (M) was synthesized following the so-called "atrane route", using CTABr as the structure-directing agent and a molar ratio fixed to 7TEAH $3: 2 T E O S: 0.52 \mathrm{CTABr}: 0.5 \mathrm{NaOH}: 180 \mathrm{H}_{2} \mathrm{O}$. The procedure consisted in adding $\mathrm{CTABr}$ to a solution of $\mathrm{TEAH}_{3}$ and $\mathrm{NaOH}$ containing TEOS at $118{ }^{\circ} \mathrm{C}$. After dissolving $\mathrm{CTABr}$ in the liquor, water was slowly added with vigorous stirring at $70{ }^{\circ} \mathrm{C}$ to form a white suspension. This mixture was aged at room temperature overnight.

Nanoparticulated MCM-41 (N) was synthesized using the following procedure: $\mathrm{NaOH}$ was added to the $\mathrm{CTABr}$ solution, followed by adjusting the solution temperature to $95^{\circ} \mathrm{C}$. TEOS was then added dropwise to the surfactant solution. The mixture was allowed to stir for $3 \mathrm{~h}$ to give a white precipitate.

UVM-7 (U) was synthesised using, once again, the "atrane route". The molar ratio of the reagents in the mother liquor was fixed at 7TEAH $3: 2 \mathrm{TEOS}: 0.52 \mathrm{CTABr}: 180 \mathrm{H}_{2} \mathrm{O}$. The TEOS/TEAH 3 mixture was heated to $120^{\circ} \mathrm{C}$ until no elimination of ethanol was observed. The mixture was cooled to $90{ }^{\circ} \mathrm{C}$ and the $\mathrm{CTABr}$ was added gradually in small portions, followed by water. The mixture was aged for $24 \mathrm{~h}$.

The SBA-15 (S) sample was synthesized using P123 as the structure-directing agent with the reactant molar ratios: 0.017P123:1.0TEOS:6 $\mathrm{HCl}: 196 \mathrm{H}_{2} \mathrm{O}$. The preparation was carried mixing an aqueous solution of $\mathrm{P} 123$ with $\mathrm{HCl}$ solution, and stirring for $2 \mathrm{~h}$, after which the silica source, TEOS, was added. This final mixture was stirred for a further $20 \mathrm{~h}$. 
After the synthesis, the different solids were recovered, washed with deionised water,

141 and air-dried at room temperature. The as-synthesized solids were calcined at $550{ }^{\circ} \mathrm{C}$ using an

142 oxidant atmosphere for $5 \mathrm{~h}$ in order to remove the template phase.

143 The particles were also functionalised with $N^{1}$-(3-trimethoxysilylpropyl)diethylenetriamine

144 (N3). In particular, $1 \mathrm{~g}$ of the different MSPs were suspended in $40 \mathrm{~mL}$ of acetonitrile and an excess of N3 (4.3 mL, 15.0 moll g $\left.{ }^{-1}\right)$ was then added. Final mixtures were stirred for $5.5 \mathrm{~h}$ at room temperature. Finally, the solids were filtered off, washed with $30 \mathrm{~mL}$ of deionised water, and dried at room temperature.

\subsection{Simulated digestion procedure}

An in vitro digestion model consisting of mouth, gastric and intestinal phases described by Versantvoort et al. was used to simulate the typical chemical composition, $\mathrm{pH}$ and residence time periods of each of the three main compartments of the GIT [21]. A schematic representation of the in vitro digestion model is presented in Figure 1. The $\mathrm{pH}$ values of the digestive juices were checked and, if necessary, adjusted to the appropriate interval with $\mathrm{NaOH}(1 \mathrm{M})$ or $\mathrm{HCl}(37 \% \mathrm{w} / \mathrm{w})$. 


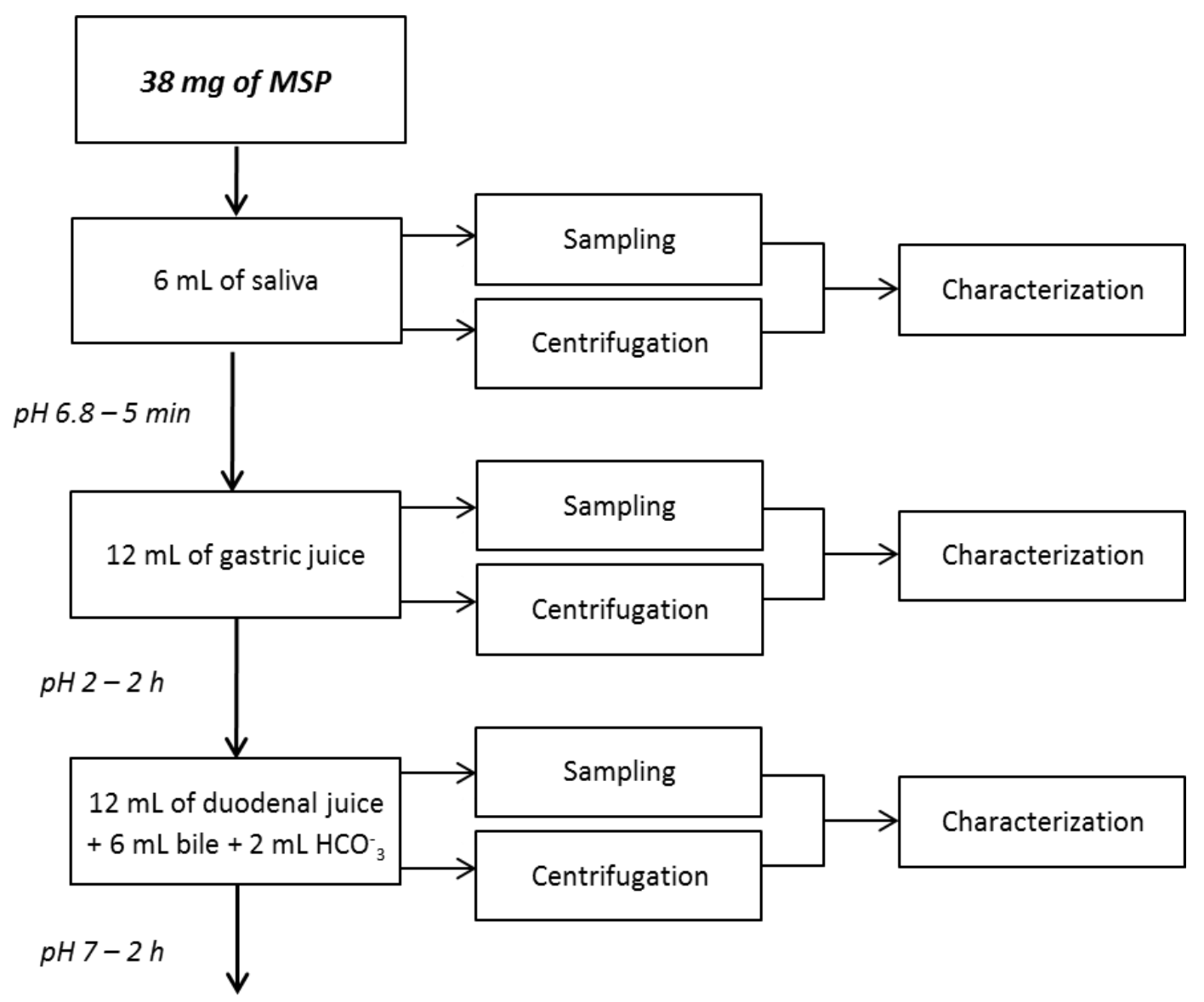

Figure 1. Schematic representation of the in vitro digestion process. The in vitro digestion model describes a threestep procedure simulating the digestive processes in mouth, stomach and small intestine. In each compartment, the matrix is incubated at $37{ }^{\circ} \mathrm{C}$ for a time relevant for the compartment. The digestion is initiated by addition of artificial saliva to the material. Subsequently, gastric juices and intestinal fluids are added to simulate the digestive processes in stomach and small intestine, respectively. After each of these steps, samples were taken to conduct characterization procedures. Characterization typically involves microscopy, PXRD, size distribution, zeta potential, $\mathrm{N}_{2}$ adsorption-desorption isotherms, ${ }^{29} \mathrm{Si} \mathrm{RMN}$, silicon analysis and biocompatibility using WST-1 test.

\subsection{Characterization of the mesoporous silica particles}

All materials, as synthetized and after a simulated digestion process, were characterized by standard procedures: i.e. X-ray diffraction (XRD), $N_{2}$ adsorption-desorption isotherms, transmission electron microscopy (TEM), field emission scanning electron microscopy (FESEM), 
confocal laser scanning microscopy (CLSM), ${ }^{29} \mathrm{Si}$ nuclear magnetic resonance (NMR), particle size distribution and zeta potential measurements.

XRD were performed on a Bruker D8 Advance diffractometer (Bruker, Coventry, UK) using CuK $\alpha$ radiation. $\mathrm{N}_{2}$ adsorption-desorption isotherms were recorded with a Micromeritics ASAP 2010 automated sorption analyser (Micromeritics Instrument Corporation, Norcross, USA). The samples were degassed at $120{ }^{\circ} \mathrm{C}$ in vacuum overnight. The specific surface areas were calculated from the adsorption data in the low pressure range using the BET model. Pore size was determined following the $\mathrm{BJH}$ method.

The effect of digestion on single particle surface and morphology was evaluated through electron microscopy observations. TEM images were obtained with a JEOL JEM-1010 (JEOL Europe SAS, Croissy-sur-Seine, France). FESEM images were acquired with a Zeiss Ultra 55 (Carl Zeiss NTS GmbH, Oberkochen, Germany) and observed in the secondary electron mode.

To determine the changes of MSPs structures and aggregation state along the whole in vitro digestion procedure, confocal laser scanning microscopy (CLSM) micrographs were taken. Three aliquots were taken from the digestion solution at $5 \mathrm{~min}, 2$ and $4 \mathrm{~h}$, just after finishing the mouth, stomach and intestine steps respectively. Samples were stained with $20 \mu \mathrm{L}$ of rhodamine $\mathrm{B}$ solution $\left(2 \mathrm{~g} \mathrm{~L}^{-1}\right)$. The observations were made $10 \mathrm{~min}$ after the dyes were added. Pictures were acquired using a Nikon Eclipse E800 microscope (Nikon, Tokyo, Japan). An Ar laser line $(488 \mathrm{~nm})$ was used to excite the rhodamine B. The images were obtained and stored at a 1,024 × 1,024-pixel resolution using the microscope software (EZ-C1 v.3.40, Nikon, Tokyo, Japan).

Microscopic studies were completed by determining the particle size distribution and surface charge at the interface of different MSPs during the in vitro digestion process. To avoid interference of organic components (sugars, enzymes, etc.) in measured values, digestive fluids addressed to determination of size distribution and zeta potential were prepared with only the inorganic components [22]. Size distribution of MSPs after each of the digestion steps 
196

197

(mouth, stomach and intestine) was measured by means of Laser Diffraction (LD) and Dynamic Light Scattering (DLS). LS determinations were performed with a Malvern Mastersizer 2000 (Malvern Instruments, Malvern, UK). For data evaluation, an optical model based on the Mie theory was created using the instrumental software assuming 1.45 as the real and 0 as the imaginary part of the refractive index of the particles. DLS determinations were performed in a Zetasizer Nano ZS equipment (Malvern Instruments, Malvern, UK). The surface charge at the interface of the MSPs or zeta potential $(\zeta)$, after each of the in vitro digestion steps, was measured at $25^{\circ} \mathrm{C}$ in a Zetasizer Nano ZS equipment (Malvern Instruments, Malvern, UK). The zeta potential was calculated from the particle mobility values by applying the Smoluchowski model. The average of five recordings was reported as zeta potential. The measurements were performed at $25^{\circ} \mathrm{C}$. Zeta potential distributions were obtained by averaging 3 measurements.

${ }^{29}$ Si MAS NMR spectra were recorded on a Varian Unity 300 spectrometer operating at 79.5 $\mathrm{MHz}$, with a magic angle spinning speed of at least $4.0 \mathrm{kHz}$. Comparison of the ${ }^{29} \mathrm{Si} \mathrm{NMR}$ spectra before and after digestion allowed evaluating the local degradation of the silica surface through rupture of S-O-Si siloxane bonds and the subsequent increment in the proportion of Si-OH terminal silanol groups.

\subsection{Quantification of silicon content in digestion fluids}

For the determination of the free silicon generated during the digestion process, digestion juices were centrifuged (9500 rpm; $10 \mathrm{~min}$ ) to separate digested MSPs from the aqueous solution. The degraded silicon concentration in the supernatant was determined by inductively coupled plasma optical emission spectrometry (ICP-OES) using a Horiba Jobin Yvon ULTIMA 2 spectrometer (Longjumeau, France). Before the analysis, the recovered supernatants were chemically digested with nitric acid under reflux. 


\subsection{WST-1 Cell viability Assays}

HeLa human cervix adenocarcinoma and HEPG2 human liver carcinoma were grown in DMEM supplemented with 10\% FBS. HCT116 human colon carcinoma cells were grown in McCoy's 5a Medium Modified supplemented with 10\% FBS and HK2 homo sapiens kidney papilloma cells were grown in Keratinocyte Serum Free Medium supplemented with bovine pituitary extract (BPE) and human recombinant epidermal growth factor (EGF). All cells were purchased from the German Resource Centre for Biological Materials (DSMZ). Cells were maintained at $37{ }^{\circ} \mathrm{C}$ in an atmosphere of $5 \%$ carbon dioxide and $95 \%$ air and underwent passage twice a week.

Cells were placed in 96-well plates at a density of 1000 cells per well. After $24 \mathrm{~h}$, plates were incubated with the digestion fluids containing MSPs at different concentrations at $37^{\circ} \mathrm{C}$ for $24 \mathrm{~h}$. After removing the digestive medium containing the MSPs, MTT solution (200 mL, 1 $\mathrm{mg} \mathrm{mL}^{-1}$ ) was added and the cells were incubated for another $3 \mathrm{~h}$. Upon removal of the MTT solution, the purple formazan crystals were solubilized with DMSO $(200 \mathrm{~mL})$ and measured at $560 \mathrm{~nm}$ on a microplate reader (SPECTRAmax plus, Molecular Devices, Sunnyvale, CA). The cytotoxicity was expressed as percentage of cell viability.

\section{Results and discussion}

\subsection{Preparation and characterization of the mesoporous silica supports}

Four mesoporous silica particles differing in size, shape, and pore structure were prepared following the procedures described by previous reports (vide ante). $\mathbf{M}$ are porous irregular-shaped particles of ca. $1 \mu \mathrm{m}$ with a 2D-hexagonal pore arrangement and pore size in the $2-3 \mathrm{~nm}$ range. With the same porous structure, $\mathbf{N}$ are spherical particles of ca. $100 \mathrm{~nm} . \mathbf{U}$ support are porous nanoparticles organized in the form of clusters creating a bimodal system of pores (mesopores and structural micropores). Finally, $\mathbf{S}$ are elongated particles of ca. $1 \mu \mathrm{m}$ 
with a well-defined hexagonal distribution of mesopores ranging from 5-8 $\mathrm{nm}$. These particles were used as models to evaluate the effect of human digestion on the stability of MSPs as well as to assess the cytotoxicity of digested MSPs in four different cell lines.

After the synthesis, the prepared MSPs were characterised using standard procedures. The mesostructure of different supports was characterised by X-Ray diffraction. As expected, the four mesoporous supports exhibited typical diffraction patterns. Figure $\mathbf{2} \mathbf{a}$ and $\mathbf{2} \mathbf{b}$ show the diffractograms of $\mathbf{M}$ and $\mathbf{N}$ solids, respectively. Curves (i) for both particles display the hexagonal ordered array of the MCM-41 family characterized by the presence of three typical peaks, indexed as (100), (110) and (200) Bragg reflections [4]. Similarities in peaks positions and full width at half maximum between the two curves confirm that the change in the particle size (nano or micro) does not have any influence in the porous structure of the particles.

Likewise, Figures $\mathbf{2} \mathbf{c}$ and $\mathbf{2} \mathbf{d}$ show typical diffractograms of $\mathbf{S}$ and $\mathbf{U}$ solids, respectively [4]. Curve (i) of figure $\mathbf{2 c}$ shows the typical pattern of $\mathbf{S}$ solid, characterised by a sharp peak at $2 \theta=1.0$, indexed as the (100) reflection, and two minor peaks in the 1.0-2.0 interval, indexed as (110) and (200) Bragg reflections, respectively. On the other hand, curve (i) of figure $\mathbf{2 d}$ presents two broad low-angle reflections that is most likely related with a disordered hexagonal array of the mesopores in the $\mathbf{U}$ support. Moreover, in all cases the functionalization of the particles with N3 (curves iii) does not modify the typical porosity of the mesoporous scaffolds, since the (100) peak is present in all cases. 

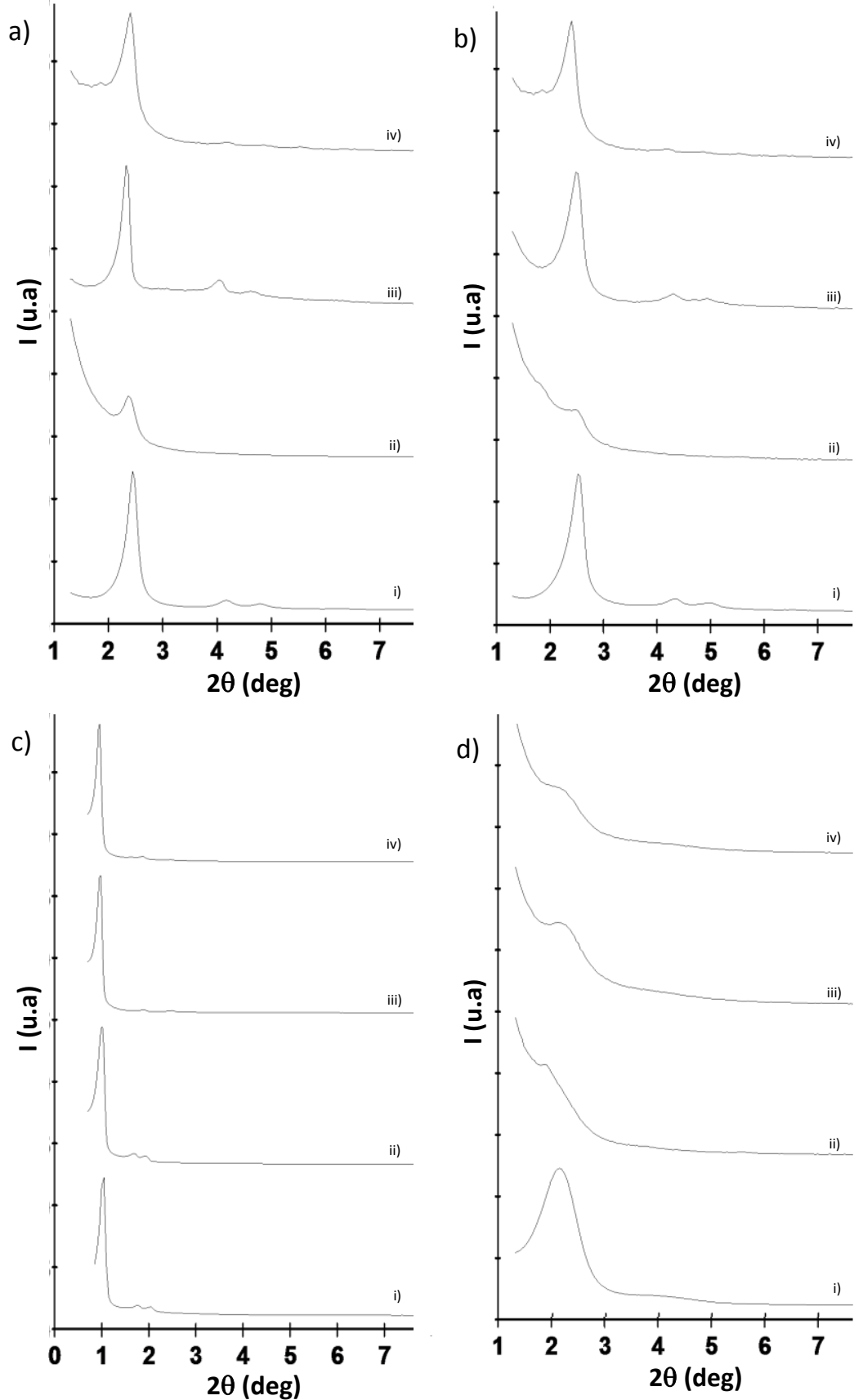

Figure 2. Powder X-ray patterns of as-synthesised MSPs before (i) and after the in vitro digestion (ii) as well as amine-functionalised MSPs before (iii) and after (iv) the in vitro digestion. MCM-41 microparticles (M) (a), MCM-41 nanoparticles (N) (b), SBA-15 (S) (c) y UVM-7 (U) (d) supports. 

desorption isotherms. Table 1 list BET specific surface values, pore volumes, and pore sizes of the particles object of study. As observed, before the digestion process, all the synthesized bare particles exhibited large pore volumes and large surface areas due to the presence of the mesopores.

Table 1. BET specific surface values, pore volumes and pore sizes calculated from the $N_{2}$ adsorption-desorption isotherms for selected materials. MCM-41 micro (M), MCM-41 nano

\begin{tabular}{cccccc}
\hline & Particle size & $S_{\mathrm{BET}}\left(\mathrm{m}^{2} \mathrm{~g}^{-1}\right)$ & Pore volume $^{a}$ & Pore size $^{a, b}(\mathrm{~nm})$ & Wall thickness $^{\mathrm{c}}$ \\
& $(\mu \mathrm{m})$ & & $\left(\mathrm{cm}^{3} \mathrm{~g}^{-1}\right)$ & & $(\mathrm{nm})$ \\
\hline $\mathrm{M}$ & $1.1 \pm 0.2$ & 1063 & 0.95 & 2.65 & 2.05 \\
$\mathrm{~N}$ & $0.09 \pm 0.02$ & 1070 & 0.92 & 2.53 & 2.91 \\
$\mathrm{~S}$ & $1.2 \pm 0.2$ & 642 & 0.93 & 7.82 & 2.22 \\
$\mathrm{U}$ & $1.2 \pm 0.3$ & 981 & 0.72 & 2.62 & 2.26 \\
\hline
\end{tabular}

\subsection{Effect of in vitro digestion in microstructure of bare particles}

To test the effect of human digestion on bare MSPs stability a typical in vitro digestion procedure was followed (Figure 1). After the digestion process, particles were washed with water, recovered by centrifugation and characterized.

The XRD diffractograms (Fig 2) revealed an important loss of the order in all bare digested particles (ii) characterized by a decreased intensity of the (100) Bragg peak and a loss of almost the rest of reflections when compared with undigested ones (i). The loss of intensity followed 
the order $\mathbf{N}$ (wall thickness $=1.91$ ), closely followed by $\mathbf{M}$ (wall thickness $=2.05$ ) and $\mathbf{U}$ (wall thickness $=2.22$ ). The differences in wall thickness among $\mathbf{N}$ (less than $2 \mathrm{~nm}$ ) and $\mathbf{M}$ and $\mathbf{U}$ (more than $2 \mathrm{~nm}$ ) might be due to the synthesis route. $\mathbf{N}$ and $\mathbf{U}$ follow the atrane route of synthesis, a procedure that provides wide framework walls. For its part, $\mathbf{S}$ with a wall thickness of $2.56 \mathrm{~nm}$ seemed to be the particle that better conserve the hexagonal structure of the particle. In fact the diffractogram of digested $\mathbf{S}$ was very similar than that of the undigested particle meaning that mesoporous arrangement is preserved during the in vitro digestion procedure. In short, the pore wall thickness seems to be the key factor to preserve the mesostructure integrity, being the silica stability highly favoured when the pore wall thickness increases.

Nitrogen sorption data (Fig 3 ) show a reduction of the adsorbed $\mathrm{N}_{2}$ volume in all solids, which suggested that the digestion procedure provokes a loss of specific surface area and pore volume. This reduction is more marked in the case of $\mathbf{N}\left(177 \mathrm{~m}^{2} \mathrm{~g}^{-1 ;} 0.29 \mathrm{~cm}^{3} \mathrm{~g}^{-1}\right)$ and $\mathbf{U}\left(372 \mathrm{~m}^{2}\right.$ $\left.\mathrm{g}^{-1 ;} 0.78 \mathrm{~cm}^{3} \mathrm{~g}^{-1}\right)$ that in $\mathbf{M}\left(321 \mathrm{~m}^{2} \mathrm{~g}^{-1 ;} 0.46 \mathrm{~cm}^{3} \mathrm{~g}^{-1}\right)$ and $\mathbf{S}\left(368 \mathrm{~m}^{2} \mathrm{~g}^{-1 ;} 0.62 \mathrm{~cm}^{3} \mathrm{~g}^{-1}\right)$ in consonance with XRD patterns. This loss of surface area and pore volume as a consequence of the contact with biological media has also been observed by other authors $[15,18]$. In these works, the loss of textural properties of silica supports was associated with a progressive elimination of the porosity by dissolution of the silica or by pore blockage due to precipitation of inorganic compounds onto the surface of the porous silica. The combined result of silica redisolution and salt precipitation has a marked effect on the form of the isotherms. In fact, only SBA-15 shows after digestion a $\mathrm{N}_{2}$ adsorption-desorption isotherms qualitatively similar to the original one, with a well-defined adsorption step at relative pressure values in the 0.6-0.8 range. In the case of the remaining silica supports with thicker walls ( $\mathbf{M}$ and $\mathbf{U}$ ), the loss of surface area and volume seems to be more pronounced than the mesostructural disorder evidenced through XRD. At this point, probably the existence of small mesopores for samples $\mathbf{M}, \mathbf{N}$ and $\mathbf{U}$ (ca. 2.5 $\mathrm{nm}$ ) leads to an easier pore blocking through salt reprecipitation with the subsequent 
a)

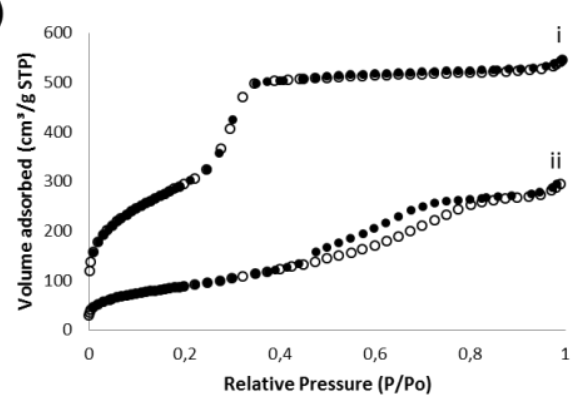

c)

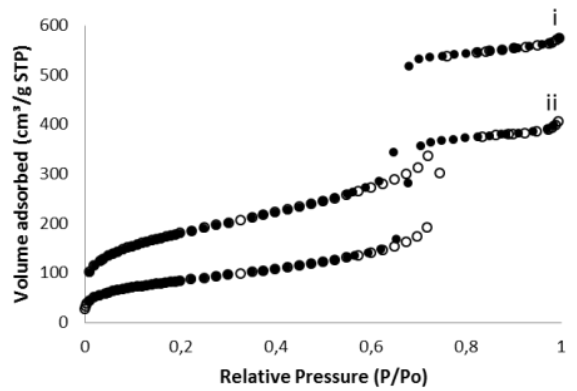

b)

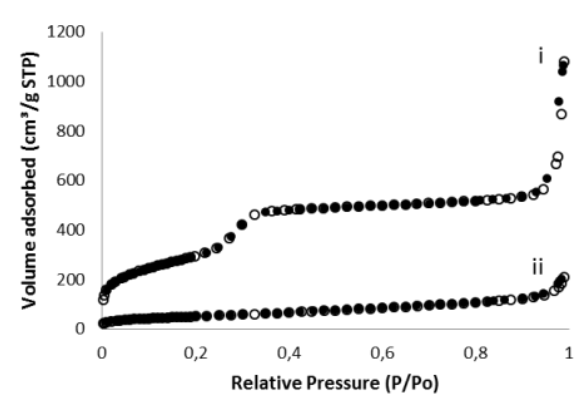

d)

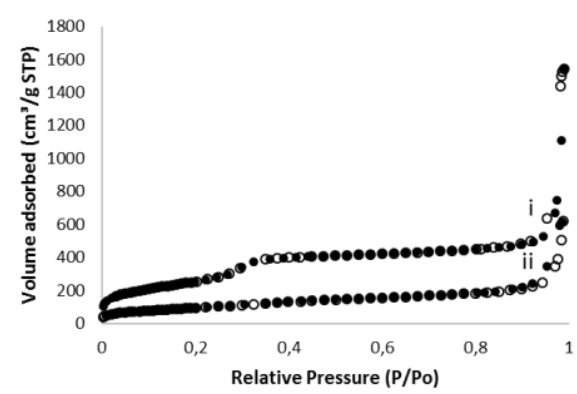

323

Figure 3. Nitrogen adsorption-desorption isotherms for microparticulated MCM-41 (M) (a), nanoparticulated MCM41 (N) (b), SBA-15 (S) (c) and UVM-7 (U) (d) before (i) and after (ii) the in vitro digestion procedure.

To further understand which of these mechanisms are involved in the digestion-induced silica degradation, in parallel to XRD and $\mathrm{N}_{2}$ absorption-desorption isotherms experiments, TEM and FESEM observations of the four silica supports were carried out. Figure 4 shows FESEM and TEM pictures of different bare particles before and after the in vitro digestion process. This figure allows observing not only the particle size and shape of the single particle, but also particle porosity.

After the digestion, two particles did not modify their appearance (FESEM) and pore integrity (TEM). These particles are $\mathbf{M}$ and $\mathbf{S}$ which are particle with size in the microscale. This implies that for these particles the loss of order observed in XRD was not provoked by a collapse of the mesostructure, but probably by the formation of a small volume fraction of 
337 "gel" consisting mostly of $\left(\mathrm{SiOH}_{2}\right)_{\mathrm{n}}{ }^{18}$ or the adsorption of calcium and phosphate ions present

338 in the digestion fluids on the silica surface forming a hydroxyapatite phase [15, 23-25]. In contrast, supports based on nanoparticulated materials, $\mathbf{N}$ and $\mathbf{U}$ biodegraded with a modification of the appearance of both, surface and pore structure as a consequence of the digestion procedure. Concretely, the most affected support was N. After the whole digestion process, $\mathbf{N}$ and $\mathbf{U}$ nanoparticles lost the uniformity of pore structure (see TEM images) and despite keeping its particle size and shape, there is a clear alteration of the surface (FESEM). In those cases, besides the pore closure provoked by the apparition of new phases, the decrease of the mesostructure observed by XRD and $\mathrm{N}_{2}$ adsorption-desorption isotherms can also be originated, at least partially, by pore collapse. 
FESEM of MSP

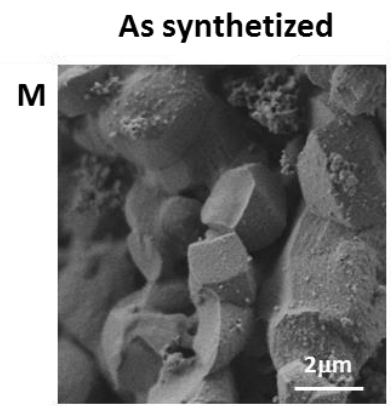

N

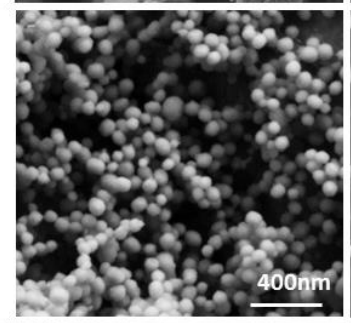

S

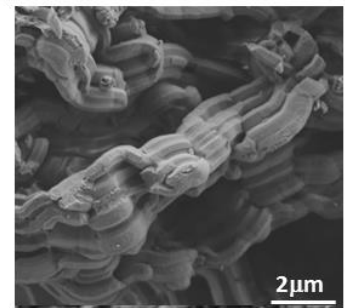

$\mathbf{U}$

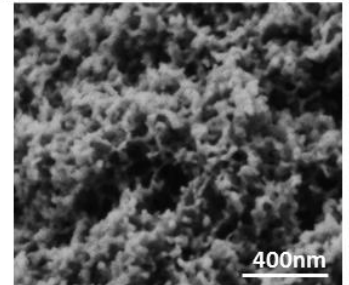

After the IDP
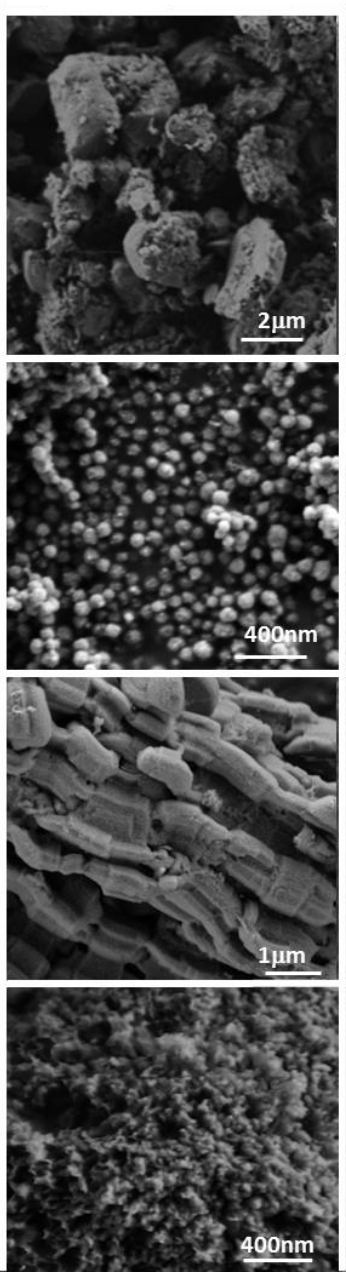

TEM of MSP dispersed in dichloromethane

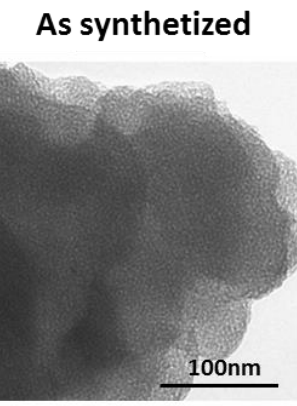

After the IDP
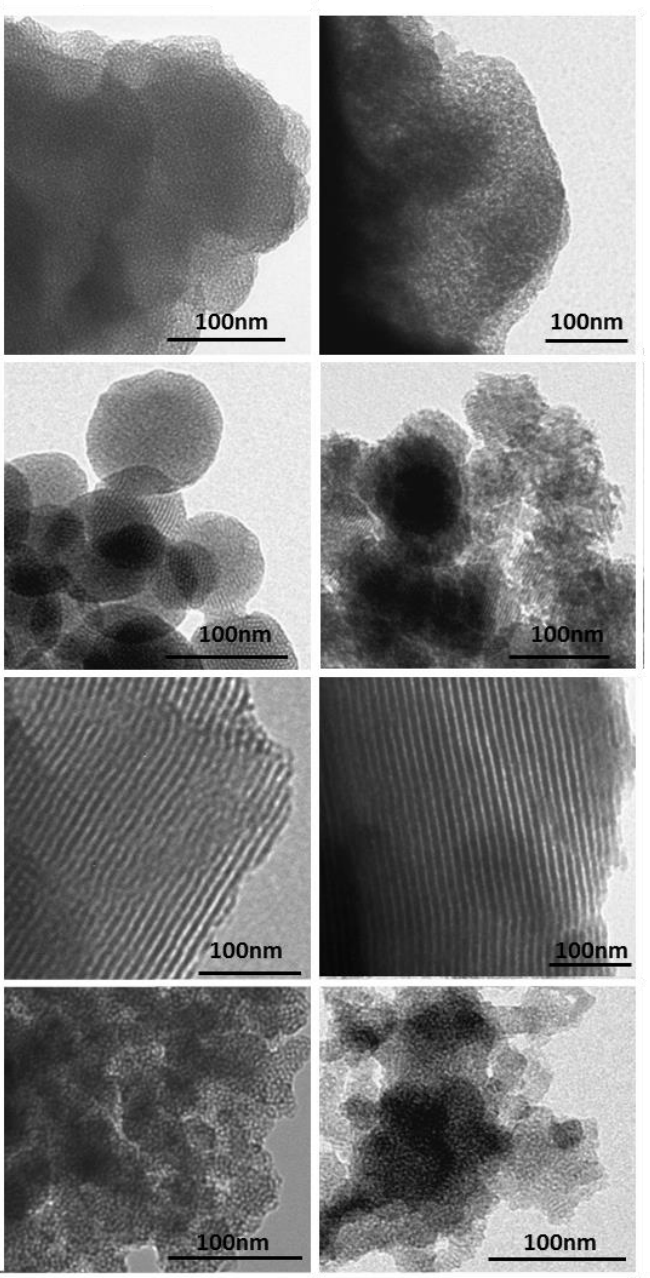

Figure 4. Characterization of particle size, particle shape and pore system of bare MSPs before and after the in vitro digestion procedure (IDP). MCM-41 (M), MCM-41 nano (N) SBA-15 (S) and UVM-7 (U).

Silica degradation necessarily implies the break of siloxane bonds with the subsequent generation of silanol groups and this was corroborated through the evolution of the ${ }^{29} \mathrm{Si} \mathrm{NMR}$ spectra before and after digestion. For this study we selected two samples ( $\mathbf{S}$ and $\mathbf{N}$ ) that can be considered as representative of the two observed behaviours with low (solid $\mathbf{S}$ ) and high

356 (solid $\mathbf{N}$ ) biodegradation according to TEM images. The ${ }^{29} \mathrm{Si}$ NMR spectra are shown in Figure 5.

While in the case of sample $\mathbf{S}$, the digestion does not affect the proportion of Q4:Q3:Q2

(68:30:2) sites, a decrease of the Q4 sites from $65 \%$ to $60 \%$ is observed in the case of the $\mathbf{N}$ 

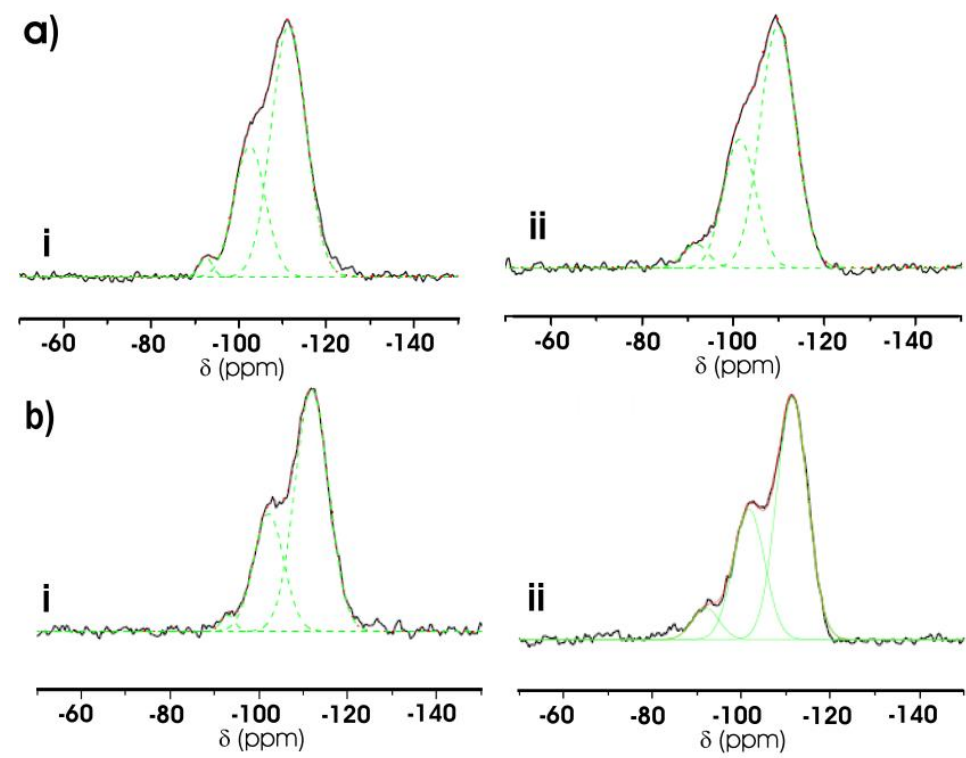

Figure 5. ${ }^{29}$ Si NMR spectra for SBA-15 (a) and nanoparticulate MCM-41 (b), before (i) and after (ii) the in vitro digestion procedure.

Having in mind these results, it is apparent that all studied MSPs are altered as a consequence of the in vitro digestion process. However, the degradation degree depends on the type and size of the particles. In this line, El Mourabit et al. studied the structure alteration of several porous silica supports differing in particle size, particle shape, pore-size distribution, specific surface area, pore volume and average of pore diameter caused by immersion in acid solutions and found that the degradation of the supports was not obviously influenced by textural properties of the particles [18]. Nevertheless, in our study, it seems to be clear that particle size and wall thickness seem to be essential parameters that condition degradation.

In order to correlate the impact of each of the phases of the digestion with the particle's degradation, a further experiment was done. For this MCM-41 nanoparticles (solid $\mathbf{N}$ ) were selected given that this was the most affected support by the whole digestion process. For this purpose, $\mathbf{N}$ was put in contact with water for $\mathbf{4} \mathrm{h}$. In parallel, a typical in vitro digestion process 
Figure 6 shows TEM micrographs of $\mathbf{N}$ after $4 \mathrm{~h}$ in contact with water (a) and after each of the phases of the in vitro digestion process: buccal (b), gastric (c) and intestinal (d). As observed, the particle size (ca. $100 \mathrm{~nm}$ ) did not vary along the digestion suggesting that particle structure remains unaltered after the whole digestion process. Moreover, surface and porosity of MCM41 remained unchanged after $4 \mathrm{~h}$ in water, meaning that particles do not collapse easily in water solution. Particles are also intact after the $5 \mathrm{~min}$ of contact with simulated saliva. However, particles change dramatically after the $2 \mathrm{~h}$ of gastric phase. In particular, after this digestion step, particles loss clearly their spherical shape and ordered porous conformation and become irregular shaped spheres with disordered porosity. Little differences among particles observed after gastric and after both, gastric and intestinal phases were observed suggesting that once the digestive solution is neutralized by the addition of intestinal juices, the degradation process stopped.

Water

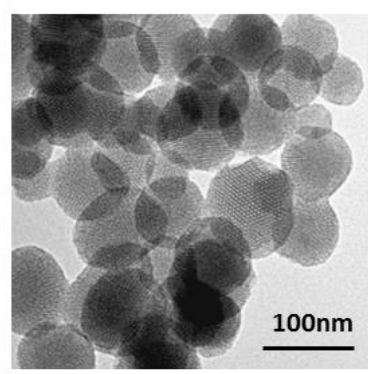

Buccal phase

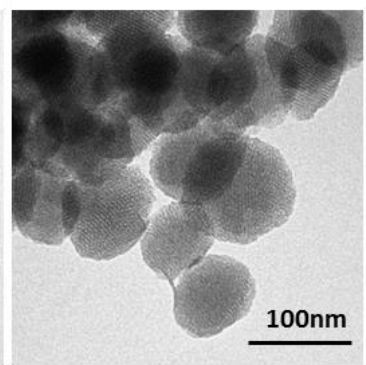

Gastric phase

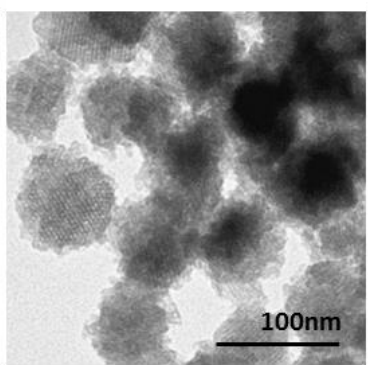

Intestinal phase

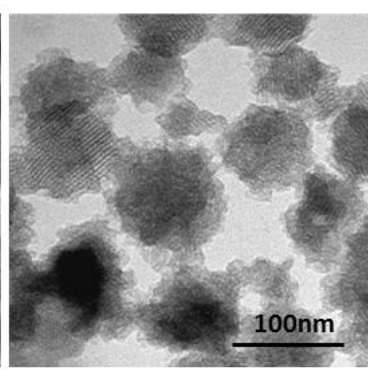

Figure 6. TEM images showing particle size, particle shape and pore system of bare MCM-41 nanopaticles (solid $\mathbf{N}$ ) after $4 \mathrm{~h}$ in water, and buccal, gastric and intestinal phases of the in vitro digestion procedure.

These findings confirm that gastric phase $(\mathrm{pH} 2)$ is the responsive of particle's degradation. The role of acids in porous silica degradation has previously been described [18]. These authors realised that protons play a role in the acidic alteration process of silica. Moreover, they pointed out that in acidic conditions, anions (i.e. $\mathrm{SO}_{4}{ }^{2-}, \mathrm{Cl}^{-}, \mathrm{PO}_{4}{ }^{3-}$ ) present in the media can act as nucleophilic catalysts accelerating the degradation reactions. Having in mind the 
composition of the two fluids comprising gastric phase of the digestion (i.e. saliva and gastric juice) it can be say that all these degradative species (i.e. $\mathrm{NaSO}_{4}, \mathrm{NaH}_{2} \mathrm{PO}_{4}, \mathrm{HCl}, \mathrm{NaCl}, \mathrm{KCl}, \mathrm{CaCl}_{2}$ and $\mathrm{NH}_{4} \mathrm{Cl}$ ) are present in our studies.

\subsection{Effect of in vitro digestion in microstructure of amine-functionalised particles}

To investigate if the functionalization of the surface of the particles with certain organic molecules have any influence in the preservation of the structure during the in vitro digestion, the four particles object of study were functionalised with $N^{1}$-(3trimethoxysilylpropyl)diethylenetriamine. This polyamine is one common organic molecule used to prepare capped mesoporous silica particles able to modulate payload release in response to $\mathrm{pH}$ changes [26]. After functionalization, particles were subjected to the digestion process described in Figure 1. Digested particles were washed with water, dried and characterized by XRD, TEM and FESEM.

Figure 2 shows XRD patterns of amine-functionalized particles before (ii) and after (iv) the in vitro digestion process. In contrast to bare particles, functionalised particles showed the same diffraction peaks before and after the digestion, which indicate a preservation of the porous structure after the digestion procedure. The structure preservation was confirmed by microscopic analysis. As observed in Figure 7, morphology, particle size and porous structure of the different amine-functionalized supports is very similar before and after the in vitro digestion process. These studies point out the role played by amines in the protection of porous silica, especially in the nanoparticles (solids $\mathbf{N}$ and $\mathbf{U}$ ), against the attack by acids and chemical species present in the digestive juices. The prevention of silica degradation after organic functionalization has also been observed by other authors. Lin et al. found that degraded Si amounts from $42 \mathrm{~nm}$ diameter silica nanoparticles were greater than that from the equivalent pegylated nanoparticles after both 10 days in deionized water and PBS at room temperature and $37^{\circ} \mathrm{C}$ [17]. Cauda et al. also observed that the attachment of a poly(ethylene 
426

427

glycol)-layer on the outer surface of colloidal mesoporous silica stabilized the particles by reducing the rate of degradation in simulated body fluid at $37{ }^{\circ} \mathrm{C}$ for 1 month [15]. The preventive effect of functional groups attached to the surface of the silica supports on the degradation of porous silica could be attributed to the capability of these molecules to inhibit the attack caused by acids and catalytic anions as well as to prevent the adsorption of calcium/phosphate cations and the rearrangement of silicon species on a new "gel" fraction on the walls of the particles.

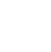

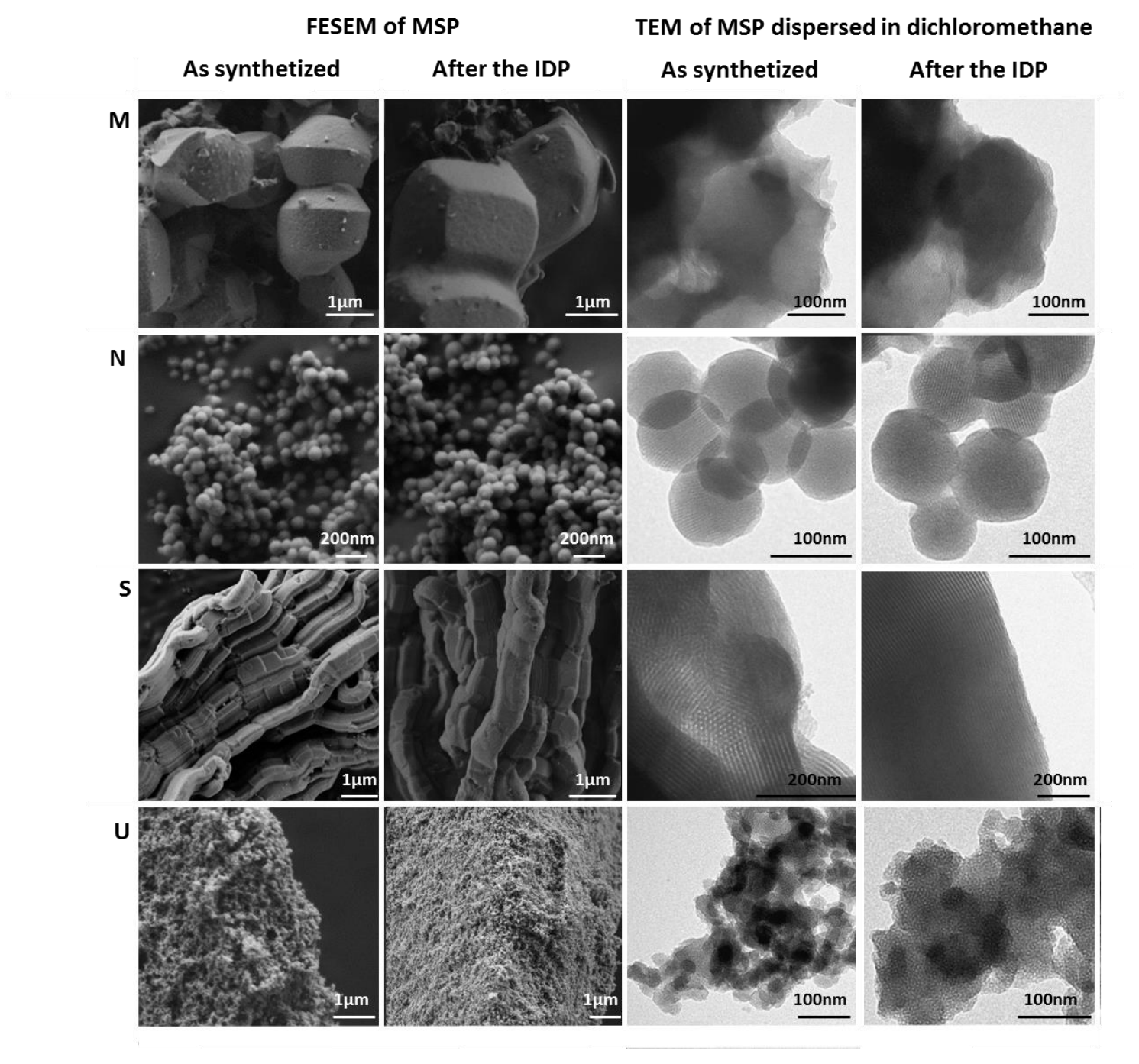

Figure 7. Characterization of particle size, particle shape and pore system of amine-functionalised MSPs before and after the in vitro digestion procedure. MCM-41 (M), MCM-41 nano (N) SBA-15 (S) and UVM-7 (U). 
In our study, according to $\mathrm{CNH}$ elemental analysis data, the post-functionalization degree of our samples was comprised in the $0.14-0.15$ mol of $\mathrm{N}^{1}$-(3-trimethoxysilylpropyl) groups per

$440100 \mathrm{~g}$ of sample. This value suppose a density of functional groups of ca. 1-1.5 groups/nm assuming that their incorporation is effective along the whole surface (external and internal). As it is well known, the length of this organic group constitutes a serious drawback to achieve a good diffusion and dispersion along the mesopores. Then, a certain accumulation and condensation of the organic groups on the external surface and in the entrance of the mesopores is expected. This relatively thick shell seems to be the responsible for the stability of the silica supports after digestion. Moreover, in our case, amine groups attached to particle's surface can also able to locally neutralize the acidic environment created by $\mathrm{HCl}$.

\subsection{Effect of in vitro digestion in macroscopic structure}

To determine the changes of MSPs structures and aggregation state along the whole in vitro digestion procedure, confocal laser scanning microscopy (CLSM) micrographs were taken upon staining $\mathbf{M}, \mathbf{N}, \mathbf{S}$ and $\mathbf{U}$ supports at different stage of the digestion with rhodamine B (Fig $\mathbf{8}$ ). This study revealed that all the particles tended to form aggregates in water. This trend to form large aggregates, especially observed in $\mathbf{N}$ and $\mathbf{U}$, is in accordance with results previously reported $[27,28]$. Figure 8 also allows observing that for all particles the gastric phase trend to 


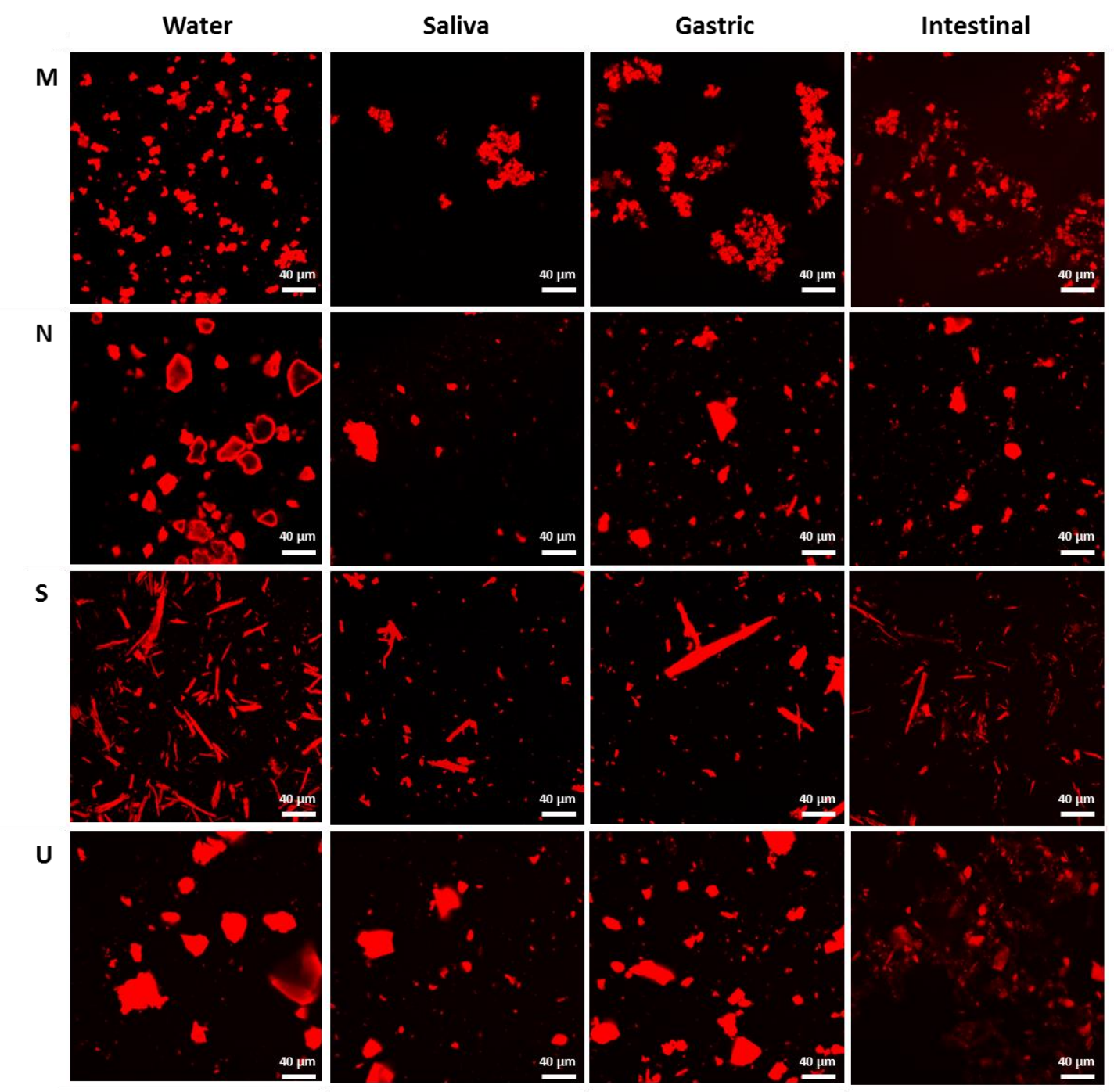

Figure 8. Characterization of particle size and particle shape of bare MSPs before and after the in vitro digestion procedure. MCM-41 micro (M), MCM-41 nano (N), SBA-15 (S) and UVM-7 (U).

Aggregation tendency observed in gastric phase for all particles was confirmed by particle size distribution measurements using light diffraction. As shown in Figure 9, according to particles' grain size in different digestive media, $\mathbf{S}$ and $\mathbf{M}$ microparticles, exhibited a size distribution in the range $0.5-2 \mu \mathrm{m}$, while size distribution of $\mathbf{N}$ and $\mathbf{U}$ ranged from 5 up to 40 $\mu \mathrm{m}$. The inset graphs of the same figure shows size distribution of nano-sized silica present in the digestion fluids. As presumable, only two samples ( $\mathbf{N}$ and $\mathbf{U}$ ) also shown particles in the 100-200 nm range, corresponding to those particles not participating in the particle's clusters. 

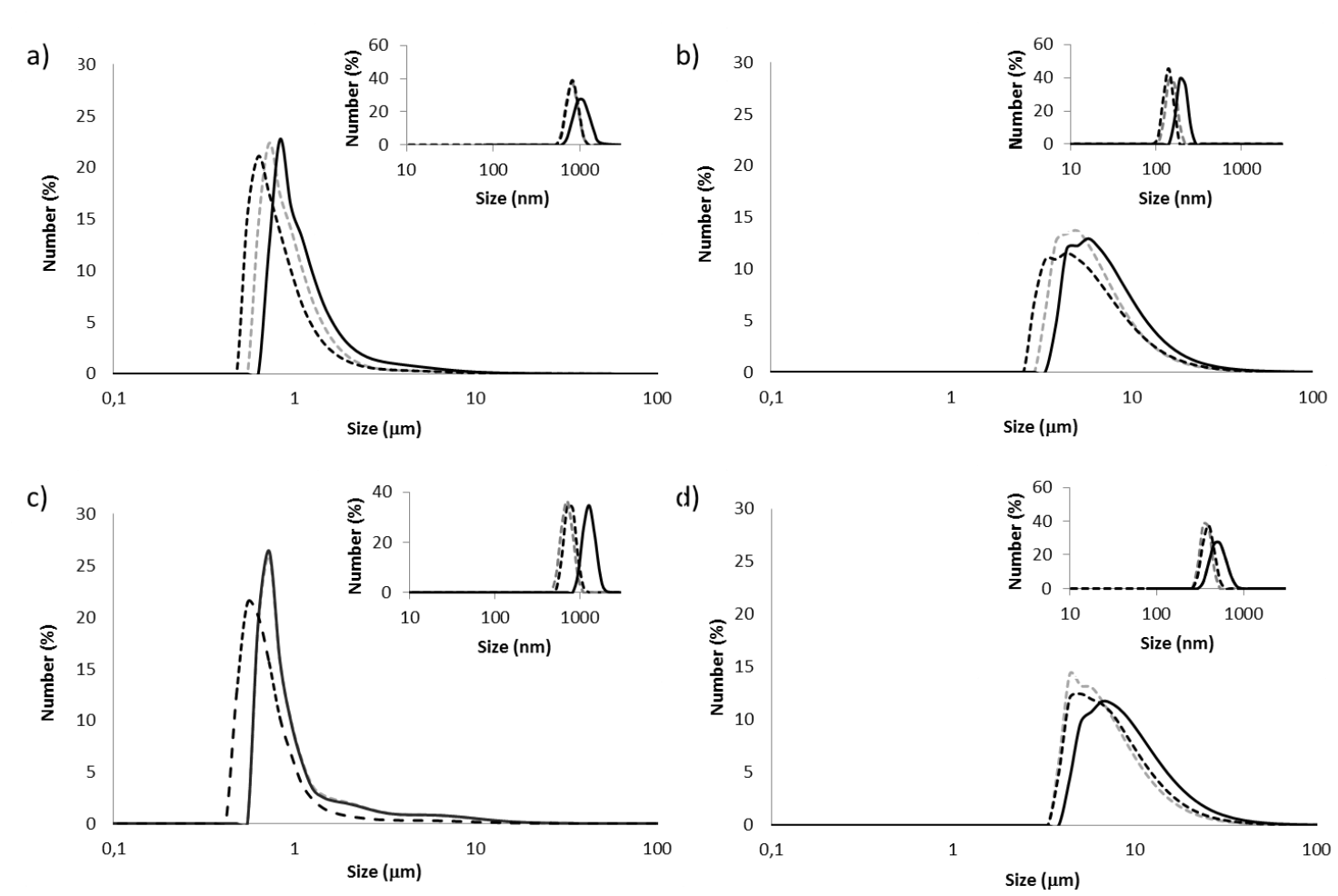

471 aggregation tendency.

Figure 9. Hydrodynamic size distribution of MCM-41 microparticles (M) (a), MCM-41 nanoparticles (N) (b), SBA-15

(S) (c) and UVM-7 (U) (d) expressed as number percentage. Discontinuous gray line (buccal), continuous black line (gastric) and discontinuous black line (intestinal).

The tendency to form large silica agglomerates at low $\mathrm{pH}$ is also supported by z-potential values and the extended DLVO theory [22]. Generally, it is considered that particles exhibiting a zeta potential in the range from +30 to $-30 \mathrm{mV}$ tend to aggregate [29]. As observed in Figure S1, particles exhibited a negative zeta potential (ca. $-30 \mathrm{mV}$ ) in the presence of saliva (neutral $\mathrm{pH})$ that changed dramatically during gastric phase $($ ca. $+10 \mathrm{mV})$ due to the protonation of silanolates at the $\mathrm{pH}$ of the stomach $(\mathrm{pH}$ 2) [30]. Moreover, after neutralization in the intestinal phase, zeta potential reached again negative values of ca $-20--30 \mathrm{mV}$, thus reducing 


\subsection{Quantification of silica degradation and degree of silica network condensation}

In previous sections, it has been concluded that bare particles, especially $\mathbf{N}$ and $\mathbf{U}$, lose part of their textural properties as a consequence of the in vitro digestion process. It was also observed, by TEM and FESEM pictures, that stomach phase was the responsive of the structural changes of the porous systems and changes in the surface appearance. To better understand the degradation process it was our aim to quantify the amount dissolved silica obtained during digestion via the quantification of free silicon by ICP-OES as an indicator of silica degradation [17]. As observed in Table S1, amounts of silicon species in solution in the digestive juice after the $4 \mathrm{~h}$ of digestion process was very similar for all particles (20-25 $\mathrm{mg} \mathrm{Si} \mathrm{L}^{-}$

${ }^{1}$ of digestive fluid) and surface functionalization with polyamines did not modify this value. These amounts of free silicon are equivalent to $4-5 \%$ of the silicon present in the starting MSPs, depending on the support. Thus, as previously indicated, these data suggested that degradation of porous structure of bare porous silica is not due to a simple dissolution of silica provoking a collapse of the particle, but by a transformation of the silica in a new disordered phase. In addition, determination of free silicon formed in the three phases of the digestion of bare MCM-41 nanoparticles (solid $\mathbf{N}$ ) revealed that only $0.4 \%$ of the silicon was dissolved during the buccal phase of digestion whereas percentages of dissolved silicon reached values of $2 \%$ in the stomach phase and continued increasing until $4 \%$ at the end of the intestinal phase.

\subsection{Biocompatibility of particles after the in vitro digestion}

In previous works, we studied the cytotoxicity of the four particles object of study which were loaded with folic acid and functionalized with $\mathrm{N}$-(3trimethoxysilylpropyl)diethylenetriamine [4]. The study concluded that functionalised $\mathbf{M}, \mathbf{N}, \mathbf{S}$ and $\mathbf{U}$ particles did not induce significant cell death upon $200 \mathrm{mg} \mathrm{mL}^{-1}$ for $24 \mathrm{~h}$. However, it has been recently reported that biodegradation of silica into silicic acids including monomeric 
510 silicic acid and various polysilicic acids with different polymerization degrees could cause

511 cytotoxicity via adsorbing and binding enzymes and substrate proteins [16].

512 In previous sections it has been demonstrated that MSPs were degraded during the digestion procedure, and that ca. 4-5\% of the silicon present in the MSPs was finally found in the solution. Thus, with the purpose of evaluate potential side effects of degradation products of the digested supports on human cells we aimed in this section to study the cytotoxicity of digestive fluids containing different concentrations of MSPs. To test cytotoxicity, human colon carcinoma cells (HCT116), human liver carcinoma cells (HEPG2), human kidney epithelial cells (HK2), and human cervix carcinoma cells (HeLa) cells were chosen. Studies were carried out with both, bare and amine-functionalised $\mathbf{M}, \mathbf{N}, \mathbf{S}$ and $\mathbf{U}$ particles after the in vitro digestion procedure described above. compromised even when exposed at different digestive fluids containing relatively large concentrations MSPs (up to $200 \mathrm{mg} \mathrm{mL}^{-1}$ ). As stated above it is considered that polysilicic acids developed during silica degradation could cause cytotoxicity via adsorbing and binding enzymes and substrate proteins. In contrast, monomeric silicic acid does not bind proteins and therefore has no cytotoxicity [16]. Having this in mind, results of this study suggested that both, the formation of silicic species is relatively low and that the biodegradation products do not contain large amounts of cytotoxic polysilicic acids with high polymerization degree.

\section{Conclusions}

The present work has studied the effect of an in vitro digestion process on the stability of bare and amine-functionalized mesoporous silica particles. Results showed that bare SBA-15 and MCM-41 microparticles were very stable against degradation. However, supports based on nanoparticles (i.e. MCM-41 nanoparticulated and UVM-7) exhibited an evident degradation 
535

536

537

538

539

540

541

542

543

544

545

546

547

548

549

550

551

552

553

554

555

556

557

558

559

560

process, only ca. $5 \%$ of the silicon present in the sample was dissolved in the digestion fluids, confirming that the degradation process is mainly based in the chemical transformation of the $\mathrm{SiO}_{2}$ in other phases and not in the loss of matter. This degradation was prevented by the functionalization of the external surface of the particles with $N^{1}$-(3trimethoxysilylpropyl)diethylenetriamine. These findings evidence the importance of particle size and surface modification on the degradation behaviour during an in vitro digestion process. In addition, despite the partial dissolution of the particles during the different phases of the digestion, neither the digested particles nor the biodegradation products show any toxicity to HCT116, HEPG2 HK2 and HeLa cells. In accordance to these results, the utilization of mesoporous silica microparticles, and over all, amine-functionalized mesoporous silica microparticles in the design of oral delivery systems guarantees the chemical stability of the supports through the whole digestive tube and no signs of toxicity have been observed.

\section{Acknowledgements}

Authors gratefully acknowledge the financial support from the Ministerio de Economía y Competitividad (Projects AGL2012-39597-C02-01, AGL2012-39597-C02-02 and MAT201238429-C04-01 and MAT2012-38429-C04-03), Generalitat Valenciana (project PROMETEO/2009/016) and FEDER founding. E.P. and M.R are grateful to the Ministerio de Ciencia e Innovación for their grants (AP2008-00620, AP2010-4369). Electron Microscopy Service of the UPV is also acknowledged.

\section{REFERENCES}

[1] J. Salonen, L. Laitinen, A. M. Kaukonen, J. Tuura, M. Björkqvist, T. Heikkilä, J. Hirvonen, V. P. Lehto, J. Controlled Release 108 (2) (2005) 362-374.

(2] F. Wang, H. Hui, T. J. Barnes, C. Barnett, C. A. Prestidge, Mol. Pharm. 7 (1) (2009) 227-236.

[3] U. Brohede, R. Atluri, A. E. Garcia-Bennett, M. Stromme, Curr. Drug. Deliv. 5 (3), (2008) 177-185. 
[4] É. Pérez-Esteve, M. Ruiz-Rico, C. de la Torre, L. Villaescusa, F. Sancenón, M. D. Marcos, P. Amorós, R. Martínez-Máñez, J. M. Barat, Food Chemistry 196, (2016) 66-75.

[5] A. Popat, S. Jambhrunkar, J. Zhang, J. Yang, H. Zhang, A. Meka, C. Yu, Chem. Comm. 50 (42) (2014) 5547-5550.

[6] D. Arcos, M. Vallet-Regí, M. Bioceramics for drug delivery, Acta Materialia, 61 (3), (2013) 890911.

[7] W. H. Suh, K. S. Suslick, G. D. Stucky, Y. H. Suh, Progress in neurobiology 87 (3) (2009) 133-170.

[8] Q. Gao, Y. Xu, D. Wu, W. Shen, F. Deng, Langmuir 26 (22) (2010) 17133-17138.

[9] P. Yang, S. Gai, J. Lin, Chem. Soc. Rev. 41 (9) (2012) 3679-3698.

[10] E. Aznar, M. Oroval, L. Pascual, J. R. Murguía, R. Martínez-Máñez, F. Sancenón, Chem. Rev. 2016 116 (2) 561-718

[11] R. Mellaerts, R. Mols, J. A. Jammaer, C. A. Aerts, P. Annaert, J. Van Humbeeck, G. Van den Mooter, P. Augustijns, J. A. Martens, Eur. J. Pharm. Biopharm. 69 (1) (2008) 223-230.

[12] S. Kalepu, V. Nekkanti, Acta Pharm. Sin. B. 5 (5) (2015) 442-453.

[13] É. Pérez-Esteve, M. Ruiz-Rico, R. Martínez-Máñez, J. M. Barat, J. Food Sci, 80 (11) (2015) E2504E2516.

[14] Q. He, Z. Zhang, Y. Gao, J. Shi, Y. Li, Small 5 (23) (2009) 2722-2729.

[15] V. Cauda, A. Schlossbauer, T. Bein, Microporous Mesoporous Mater. 132 (1) (2010) 60-71.

[16] Q. He, J. Shi, M. Zhu, Y. Chen, F. Chen, Microporous and Mesoporous Mater. 131 (1) (2010) 314320.

[17] Y. S. Lin, N. Abadeer, C. L. Haynes, Chem. Commun. 47 (1) (2011) 532-534.

[18] S. El Mourabit, G. Toquer, J. Cambedouzou, F. Goettmann, A. Grandjean, RSC Advances, 2 (2012) 10916-10924.

[19] Y. Choi, J. E. Lee, J. H. Lee, J. H. Jeong, J. Kim, Langmuir 31 (2015) 6457-6462.

[20] D. Arcos, M. Vallet-Regí. Bioceramics for drug delivery. Acta Mater. 61 (3) (2013) 890-911.

[21] C. H. Versantvoort, A. G. Oomen, E. Van de Kamp, C. J. Rompelberg, A. J. Sips, Food Chem. Toxicol., 43 (1) (2005) 31-40.

[22] R. Peters, E. Kramer, A. G. Oomen, Z. E. Rivera, G. Oegema, P. C. Tromp, R. Fokkink, A. Rietveld, H. J. Marvin, S. Weigel, A. A. Peijnenburg, H. Bouwmeester, ACS Nano 6 (3) (2012) 2441-2451. 
590

591

592

593

594

595

596

597

598

599

600

601

[23] C. Ohtsuki, T. Kokubo, T. Yamamuro, J. Non-Cryst. Solids 143 (1992) 84-92.

[24] S. B. Cho, F. Miyaji, T. Kokubo, K. Nakanishi, N. Soga, T. Nakamura, J. Mater. Sci. - Mater. Med. 9 (5) (1998) 279-284.

[25] S. Radin, S. Falaize, M. H. Lee, P. Ducheyne, Biomaterials 23 (15) (2002) 3113-3122.

[26] A. Bernardos, E. Aznar, C. Coll, R. Martínez-Mañez, J. M. Barat, M. D. Marcos, F. Sancenón, A. Benito, J. Soto, J. Controlled Release 131 (3) (2008) 181-189.

[27] E. Pérez-Esteve, L, Oliver, L. García, M. Nieuwland, H. H. de Jongh, R. Martínez-Máñez, J. M. Barat, Langmuir 30 (23) (2014) 6970-6979.

[28] Q. Zhang, F. Liu, K. T. Nguyen, X. Ma, X. Wang, B. Xing, Y. Zhao, Adv. Funct. Mater. 22 (24) (2012) 5144-5156.

[29] O. Duman, S. Tunç, Microporous Mesoporous Mater. 117 (1) (2009) 331-338.

[30] M. Kosmulski, J. Colloid Interface Sci. 208 (2) (1998) 543-545. 